\title{
RESEARCH
}

Open Access

\section{Muscle histopathology in nebulin-related nemaline myopathy: ultrastrastructural findings correlated to disease severity and genotype}

Edoardo Malfatti 1,2,3,4, Vilma-Lotta Lehtokari ${ }^{6}$, Johann Böhm7, Josine M De Winter ${ }^{8}$, Ursula Schäffer ${ }^{7}$, Brigitte Estournet ${ }^{9}$, Susana Quijano-Roy ${ }^{9}$, Soledad Monges ${ }^{10}$, Fabiana Lubieniecki ${ }^{10}$, Remi Bellance ${ }^{11}$, Mai Thao Viou', Angéline Madelaine ${ }^{1}$, Bin Wu ${ }^{12}$, Ana Lía Taratuto ${ }^{10}$, Bruno Eymard ${ }^{5}$, Katarina Pelin ${ }^{13}$, Michel Fardeau', Coen AC Ottenheijm ${ }^{8}$, Carina Wallgren-Pettersson ${ }^{6}$, Jocelyn Laporte ${ }^{7}$ and Norma B Romero $1,3,4,5^{*}$

\begin{abstract}
Nemaline myopathy (NM) is a rare congenital myopathy characterised by hypotonia, muscle weakness, and often skeletal muscle deformities with the presence of nemaline bodies (rods) in the muscle biopsy. The nebulin (NEB) gene is the most commonly mutated and is thought to account for approximately $50 \%$ of genetically diagnosed cases of NM. We undertook a detailed muscle morphological analysis of 14 NEB-mutated NM patients with different clinical forms to define muscle pathological patterns and correlate them with clinical course and genotype. Three groups were identified according to clinical severity. Group $1(n=5)$ comprises severe/lethal NM and biopsy in the first days of life. Group $2(n=4)$ includes intermediate NM and biopsy in infancy. Group $3(n=5)$ comprises typical/mild NM and biopsy in childhood or early adult life. Biopsies underwent histoenzymological, immunohistochemical and ultrastructural analysis. Fibre type distribution patterns, rod characteristics, distribution and localization were investigated. Contractile performance was studied in muscle fibre preparations isolated from seven muscle biopsies from each of the three groups. G1 showed significant myofibrillar dissociation and smallness with scattered globular rods in one third of fibres; there was no type 1 predominance. G2 presented milder sarcomeric dissociation, dispersed or clustered nemaline bodies, and type 1 predominance/uniformity. In contrast, G3 had well-delimited clusters of subsarcolemmal elongated rods and type 1 uniformity without sarcomeric alterations. In accordance with the clinical and morphological data, functional studies revealed markedly low forces in muscle bundles from G1 and a better contractile performance in muscle bundles from biopsies of patients from $\mathrm{G} 2$, and $\mathrm{G} 3$.

In conclusion NEB-mutated NM patients present a wide spectrum of morphological features. It is difficult to establish firm genotype phenotype correlation. Interestingly, there was a correlation between clinical severity on the one hand and the degree of sarcomeric dissociation and contractility efficiency on the other. By contrast the percentage of fibres occupied by rods, as well as the quantity and the sub sarcolemmal position of rods, appears to inversely correlate with severity. Based on our observations, we propose myofibrillar dissociation and changes in contractility as an important cause of muscle weakness in NEB-mutated NM patients.
\end{abstract}

Keywords: Congenital myopathies, Nemaline myopathy, Nebulin, Muscle contractility

\footnotetext{
* Correspondence: nb.romero@institut-myologie.org

'Unité de Morphologie Neuromusculaire, Institut de Myologie, Groupe

Hospitalier Universitaire La Pitié-Salpêtrière, Paris, France

${ }^{3}$ Inserm, U974, Paris F-75013, France

Full list of author information is available at the end of the article
} 


\section{Introduction}

Nemaline myopathy (NM) is a congenital muscle disorder associated with hypotonia, muscle weakness, and often skeletal muscle deformities with the presence of numerous nemaline bodies (rods) in muscle biopsy [1]. Clinically the disorder has a marked clinical variability, ranging from neonatal lethal to mild non-progressive forms with onset in childhood and adulthood. NM has been classified into six clinical categories according to the severity of the disease, the age of onset and the pattern of muscle weakness [2]. To date at least nine genes have been implicated in NM (ACTA1, MIM\#161800; NEB, MIM\#256030; TPM2, MIM\#609285; TPM3, MIM\#609284; TNNT1, MIM\#605355; KBTBD13, MIM\#609273; CFL2, MIM\#610687; KLHL4O MIM\#615340; and KLHL41) encoding proteins of the thin filament of skeletal muscle sarcomere or the Kelch domain associated proteins [3-11]. ACTA1, TPM2 and TPM3 NM is inherited both as autosomal dominant or recessive trait, with de novo dominant mutations being common in all three genes. KBTBD13 $\mathrm{NM}$ is an autosomal dominant disorder. The other five genes present autosomal recessive mode of inheritance.

Nebulin is a sarcomeric structural protein crucial for the proper assembly and function of thin filaments [12]. One molecule spans nearly the entire length of the thin filament, making nebulin one of the largest polypeptides in nature. The nebulin (NEB) gene is the most commonly mutated and is thought to account for approximately $50 \%$ of genetically diagnosed cases of NM [12]. It is composed by 183 exons of which at least 17 [13] have been shown to be alternatively spliced, giving rise to several different nebulin isoforms in skeletal muscle [14]. Molecular diagnosis has mostly been based on AHPLC and confirmed by exon Sanger sequencing which are time-consuming, laborious, and expensive [15]. Recently next generation sequencing technology in combination with microarray methodology [16] has been demonstrated to be a fast and reliable tool for analysis of large genes such as $N E B$ [15]. Patients are usually compound heterozygous for two different mutations [17]. The mechanisms leading to the alteration of muscle structure or rod formation are largely unknown.

Nemaline bodies are the pathologic hallmarks of congenital NM, even if these structures may sometimes be found associated with other conditions [18]. These are protein aggregates staining red with the modified Gomori trichrome technique. They can appear within the fibres as fine isolated/diffuse structures, compact subsarcolemmal clusters, or both [19]. On electron microscopy, nemaline bodies are electron dense and generally measure 1-7 $\mu \mathrm{m}$ in length and $0.3-2 \mu \mathrm{m}$ in width. Due to their structural continuity with the Z-disk, and their resemblance to Z-disk lattice pattern, they are considered to be lateral expansions of the Z-disk [20-22]. In the case of nebulin mutation the rod formation could be due to a defect of the nebulin C-terminal, and serine-rich (SH3) domains. Concordantly, the nebulin C-terminal region, or part of it, may extend into the Z-disk [23]. Another common histologic finding of NM is type 1 predominance or type 1 uniformity [21,22]. Based on observations from consecutive muscle biopsies done in the same patient, a substitution of type 2 to type 1 fibres has been suggested to occur with increasing age $[19,24]$. All congenital NM patients seem to present a homogeneous morphological phenotype characterised by the presence of rods and type 1 predominance. However the largest series reporting on histologic NM findings were published before the identification of specific NM genes or they included patients harbouring mutations in other genes [22,25]. For this reason it is difficult to assess the existence of specific genotype-morphological phenotype correlations in the nine genetically identified forms of NM. A systematic morphological analysis of each entity is therefore pivotal in order to reveal pathogenetic mechanisms.

With the aim of characterising different patterns of muscle involvement, defining the relationship between morphological changes, genotype, and disease severity, we describe muscle morphology and functional studies of a large cohort of clinically heterogeneous $N E B$-mutated NM patients.

\section{Material and methods \\ Patients}

Fourteen patients from 13 unrelated families from France, the French Antilles, and Argentina were included in the present study. Patients were classified into three groups according to their clinical disease severity. P4 and P5 are brothers. P1 to P5 (Group 1) presented a severe/lethal congenital myopathy leading to death in the first days of life. Their muscle biopsy was performed between 2 days and 15 days of life. P6 to P9 presented an intermediate congenital myopathy and a biopsy effectuated between 2 and 10 months (Group 2); P10 to P14 presented typical or mild (P13, and P14) nemaline myopathy and a muscle biopsy performed during childhood or adolescence/early adult life (6 months-21 years; Group 3). The clinical data of these patients were systematically retrieved and retrospectively analysed. Patients were personally examined by one of 6 of the authors. Clinical and genetic characterization of $\mathrm{P} 1, \mathrm{P} 2, \mathrm{P} 4$, and $\mathrm{P} 5$ has been previously reported $[4,15,26]$.

\section{Mutation analysis}

Patients or parents gave informed consent for the genetic analysis according to French legislation (Comité de Protection des Personnes Est IV DC-2012-1693). Genomic DNA was extracted from blood by standard methods. As nemaline myopathy $(\mathrm{NM})$ is genetically heterogeneous and 
as the immense size of the nebulin gene significantly impedes classical sequences approaches [12], we performed exome sequencing on $5 \mu \mathrm{g}$ of genomic DNA from the patients and their parents as in Böhm et al. [15].

Exome sequencing was performed at the BGI (Shenzhen) on a Hiseq 2000 (Illumina) by using the Agilent $44 \mathrm{M}$ v2 SureSelect Exon enrichment kit. Variant calling was done with the SOAP software. Variants filtering and prioritization were performed by comparison with SNP databases and with the VaRank program [27]. We discarded polymorphisms with a minor allele frequency (MAF) of more than $0.5 \%$ and excluded all variants with a frequency $<20 \%$ of the total reads for a specific position. Additionally dHPLC and Sanger sequencing was performed in 7 patients as reported in Lehtokari et al. [17]. The mutations are reported according to the coding sequence of the nebulin cDNA reference sequence NM_001164508.1, and its translation.

\section{$R T-P C R$}

RNA was extracted from muscle biopsies with TRI-Reagent (Sigma), and cDNA was reverse transcribed using the SuperScript II Reverse Transcriptase (Invitrogen) and random hexamer primers. The PCR fragments of selected cDNA regions were cloned into the pGEM-T easy vector (Promega) and transformed into E. coli DH5alpha cells. Plasmid DNA was then extracted from single colonies and Sanger sequenced.

\section{Morphological studies}

An open muscle biopsy was performed in all patients after informed consent. Age at biopsy varied from 29 weeks of adjusted gestational age to 21 years. The biopsied muscle is reported in Table 1 and was deltoid in 9 patients (P1, P2, P3, P5, P6, P9, P10, P13, and P14) and vastus lateralis in 5 (P4, P7, P8, P11, and P12). In order to make a precise and comparative study of muscle biopsy findings in Group 1 we standardized the age of new-borns calculating their 'gestational adjusted age' as described in Shichiji et al. [28] (Table 1). Samples were analysed in our research laboratory at the Myology Institute in Paris or in the Neuropathology laboratory of FLENI Institute and Garrahan Hospital in Buenos Aires, Argentina. For conventional histochemical techniques $10 \mu \mathrm{m}$ thick cryostat sections were stained with haematoxylin and eosin (H\&E), modified Gomori trichrome (mGT), Periodic acid Schiff technique (PAS), Oil red $\mathrm{O}$, reduced nicotinamide adenine dinucleotide dehydrogenase-tetrazolium reductase (NADH-TR), succinic dehydrogenase (SDH), cytochrome c oxidase (COX), and adenosine triphosphatase (ATPase) preincubated at $\mathrm{pH}$ 9.4, 4.63, 4.35. Digital photographs of each biopsy were obtained with a Zeiss AxioCam HRc linked to a Zeiss Axioplan Bright Field Microscope and processed with the Axio Vision 4.4 software (Zeiss, Germany). The fibre type pattern was determined by counting 1000 fibres from each patient in ATPase 9.4 and 4.35 reactions, and by calculating the percentage of type 1 and type 2 fibres.

Based on our experience, and the fibre type proportion reported in the literature regarding the muscle analysed, we considered type 1 fibres predominance to be present when there were more than of $60 \%$ type 1 fibre in deltoid muscles, and more than $40 \%$ in vastus lateralis muscle [29]. Fibre type distribution in G1 patients was analysed comparing the data on muscle fibre patterns during the main phases of skeletal muscle development obtained from individual with no neumuscular disorder [30,31]. Moreover, where possible, we analysed fibre type proportion in age-matched control biopsies corresponding to $\mathrm{G} 2$, and $\mathrm{G} 3$ patients.

For the analysis of the proportion of fibres with rods, 800-1000 fibres of the muscle sections of each patient were analysed, and the percentage of fibres appearing with and without rods on the total number of fibres of a muscle sections was calculated; four consecutive, non-overlapping fields were counted. In addition, a classification of the rods and their pattern was effectuated. We defined the rods as being cytoplasmic when localised mainly inside the fibres sparing the subsarcolemmal areas, scattered when they were randomly distributed in the muscle fibre, diffuse, when several small rods were distributed across the whole fibres homogenously occupying the majority of their area, central when distributed mainly in the centre of the cytoplasm, and subsarcolemmal when they were localized in a compact manner close to the fibre membrane (as clusters). We also evaluated the shape of nemaline bodies being mainly globular/ovoid, squared, or elongated.

\section{Immunohistochemistry and immunofluorescence}

Frozen muscle samples for immunohistochemical and immunofluorescence analyses were available for 7 patients (P1, P6, P7, P9, P10, P11, and P14). Myosin heavy chain fast (NCL-MHCf, Novocastra Laboratories, Newcastle Upon Tyne, United Kingdom), myosin heavy chain slow (NCL-MHCs, Novocastra Laboratories, Newcastle Upon Tyne, United Kingdom), myosin heavy chain developmental (NCL-MHCd, Novocastra Laboratories, Newcastle Upon Tyne, United Kingdom), myosin heavy chain neonatal (NCL-MHCn, Novocastra Laboratories, Newcastle Upon Tyne, United Kingdom). Antibodies were visualized using immunoperoxidase techniques [28]. Myosin alpha and beta-slow heavy chain, fast $2 \mathrm{~A}$ heavy chain, and $2 \mathrm{X}$ myosin heavy chain (BA-D5, SC-71, and 6H1, Developmental Studies Hybridoma Bank, University of Iowa, Iowa City, USA) immunofluorescence were assessed on $10-\mu \mathrm{m}$-thick cryosections over night at $4^{\circ} \mathrm{C}$. Subsequently, sections were incubated with appropriate 
Table 1 Clinical, laboratory, and genetic features of patients

\begin{tabular}{|c|c|c|c|c|c|c|c|c|c|}
\hline $\begin{array}{l}\text { Patient sex } \\
\text { current age }\end{array}$ & $\begin{array}{l}\text { Ethnic origin } \\
\text { consanguinity }\end{array}$ & $\begin{array}{l}\text { Age at onset } \\
\text { (Gestational age) }\end{array}$ & $\begin{array}{l}\text { Biopsied } \\
\text { muscle } \\
\text { Age at } \\
\text { biopsy }\end{array}$ & $\begin{array}{l}\text { Morphological } \\
\text { methods } \\
\text { (Functional } \\
\text { studies) }\end{array}$ & Clinical phenotype & $\begin{array}{l}\text { Permanent } \\
\text { mechanical } \\
\text { ventilation/age }\end{array}$ & $\begin{array}{l}\text { NEB mutation: } \\
\text { nucleotide/protein } \\
\text { change }\end{array}$ & $\begin{array}{l}\text { Effect of the } \\
\text { mutations }\end{array}$ & Reference \\
\hline $\begin{array}{l}P 1, F \\
\text { deceased } \\
10 \text { days }\end{array}$ & $\begin{array}{l}\text { French } \\
\text { Caucasian, } \\
\text { Yes }\end{array}$ & $\begin{array}{l}\text { Antenatal } \\
\text { (38 weeks) }\end{array}$ & $\begin{array}{l}\text { Deltoid } \\
2 \text { days }\end{array}$ & $\begin{array}{l}\text { IHC, IF, } \\
\text { EM (Yes) }\end{array}$ & $\begin{array}{l}\text { Group } 1 / \text { Severe congenital } \\
\text { nemaline myopathy } \\
\text { Polyhydramnios, foetal } \\
\text { akinesia. Severe global } \\
\text { hypotonia, respiratory } \\
\text { distress, arthrogryposis, } \\
\text { hip hyperlaxity, club feet } \\
\text { and dysmorphic features. }\end{array}$ & $\begin{array}{l}\text { Yes From } \\
\text { birth }\end{array}$ & $\begin{array}{l}\text { ex45; c.5574C > G; } \\
\text { p.Tyr1858Stop; int122; } \\
\text { c.19101 + 5G > A; } \\
\text { p.Leu6333_Glu6367del }\end{array}$ & $\begin{array}{l}\text { exon 45: nonsense } \\
\text { mRNA decay (by RTPCR) } \\
\text { exon 122: skipping } \\
\text { confirmed by RTPCR } \\
\text { and sequencing CDNA }\end{array}$ & $\begin{array}{l}\text { Böhm et al., } \\
\text { [15] }\end{array}$ \\
\hline \multirow[t]{2}{*}{$\begin{array}{l}\mathrm{P2}, \mathrm{F} \text {, } \\
\text { deceased } \\
\text { at } 1 \text { month }\end{array}$} & \multirow[t]{2}{*}{$\begin{array}{l}\text { French Jewish } \\
\text { (Ashkenazi) Yes }\end{array}$} & \multirow[t]{2}{*}{$\begin{array}{l}\text { Antenatal, } \\
\text { (36 weeks) }\end{array}$} & \multirow[t]{2}{*}{$\begin{array}{l}\text { Deltoid } \\
5 \text { days }\end{array}$} & \multirow[t]{2}{*}{$\begin{array}{l}\text { IHC, EM } \\
\text { (No) }\end{array}$} & \multirow{2}{*}{$\begin{array}{l}\text { Group 1/Severe congenital } \\
\text { nemaline myopathy } \\
\text { Polyhydramnios, foetal } \\
\text { akinesia. Severe global } \\
\text { hypotonia, respiratory } \\
\text { distress, arthrogryposis, } \\
\text { club feet and dysmorphic } \\
\text { features. }\end{array}$} & \multirow[t]{2}{*}{$\begin{array}{l}\text { Yes From } \\
\text { birth }\end{array}$} & $\begin{array}{l}\text { homozygous } \\
\text { deletion } \\
\text { of exon } 55\end{array}$ & \multirow[t]{2}{*}{$\begin{array}{l}\text { Ashkenazi founder } \\
\text { mutation; deletion } \\
\text { of exon } 55\end{array}$} & \multirow[t]{2}{*}{$\begin{array}{l}\text { Lehtokari et al., } \\
\text { [26] }\end{array}$} \\
\hline & & & & & & & $\begin{array}{l}\text { c.7432+ 1916_7535 + } \\
\text { 372del p.Arg2478_ } \\
\text { Asp2512del }\end{array}$ & & \\
\hline $\begin{array}{l}\mathrm{P} 3, \mathrm{M} \text {, } \\
\text { deceased } \\
\text { at } 6 \text { days }\end{array}$ & $\begin{array}{l}\text { French } \\
\text { Caucasian, } \\
\text { No }\end{array}$ & $\begin{array}{l}\text { Antenatal } \\
\text { (38 weeks) }\end{array}$ & $\begin{array}{l}\text { Deltoid } \\
6 \text { days }\end{array}$ & $\begin{array}{l}\mathrm{IHC}, \mathrm{EM} \\
(\mathrm{No})\end{array}$ & $\begin{array}{l}\text { Group 1/Severe congenital } \\
\text { nemaline myopathy } \\
\text { Polyhydramnios, foetal } \\
\text { akinesia. Severe global } \\
\text { hypotonia, respiratory } \\
\text { distress, arthrogryposis, } \\
\text { club feet, and dysmorphic } \\
\text { features. }\end{array}$ & $\begin{array}{l}\text { Yes From } \\
\text { birth }\end{array}$ & $\begin{array}{l}\text { ex86 (triplicated } \\
\text { region); c.13066delT; } \\
\text { p.Tyr4356Thrfs*8 ex110; } \\
\text { c.17535G > A; } \\
\text { p.Glu5845Glu }\end{array}$ & $\begin{array}{l}\text { exon 86: frameshift } \\
\text { mutation leading } \\
\text { to either truncation } \\
\text { or degradation. } \\
\text { exon } 110: \text { splice } \\
\text { site mutation }\end{array}$ & Present paper \\
\hline \multirow[t]{2}{*}{$\begin{array}{l}\mathrm{P} 4, \mathrm{M} \text {, } \\
\text { deceased } \\
\text { at } 5 \text { days, } \\
\text { Brother of P5 }\end{array}$} & \multirow[t]{2}{*}{$\begin{array}{l}\text { French } \\
\text { Caucasian, } \\
\text { Yes }\end{array}$} & \multirow[t]{2}{*}{$\begin{array}{l}\text { Antenatal } \\
\text { (29 weeks) }\end{array}$} & \multirow[t]{2}{*}{$\begin{array}{l}\text { Vastus } \\
\text { lateralis } \\
3 \text { days }\end{array}$} & \multirow[t]{2}{*}{$\begin{array}{l}\mathrm{IHC} \\
\text { (No) }\end{array}$} & \multirow{2}{*}{$\begin{array}{l}\text { Group 1/Severe congenital } \\
\text { nemaline myopathy } \\
\text { Polyhydramnios, foetal } \\
\text { akinesia. Severe global } \\
\text { hypotonia, absence of } \\
\text { spontaneous movements } \\
\text { at birth, respiratory distress, } \\
\text { macrosomy and macrocephaly. }\end{array}$} & \multirow[t]{2}{*}{$\begin{array}{l}\text { Yes From } \\
\text { birth }\end{array}$} & $\begin{array}{l}\text { ex177; c.24686_ } \\
\text { 24687del; p.Glu8229 } \\
\text { Glufs*18 }\end{array}$ & \multirow[t]{2}{*}{$\begin{array}{l}\text { both mutations } \\
\text { truncating/degrading }\end{array}$} & \multirow[t]{2}{*}{$\begin{array}{l}\text { Pelin et al., } \\
\text { [4] } \\
\text { Lehtokari et al., } \\
{[17]}\end{array}$} \\
\hline & & & & & & & $\begin{array}{l}\text { ex163; c.23420_ } \\
\text { 23421del; p.Arg7807 } \\
\text { Serfs*16 }\end{array}$ & & \\
\hline \multirow[t]{2}{*}{$\begin{array}{l}\mathrm{P} 5, \mathrm{M} \text {, } \\
\text { deceased } \\
\text { at } 29 \text { days, } \\
\text { Brother of } \mathrm{P4}\end{array}$} & \multirow[t]{2}{*}{$\begin{array}{l}\text { French } \\
\text { Caucasian, } \\
\text { Yes }\end{array}$} & \multirow[t]{2}{*}{$\begin{array}{l}\text { Antenatal } \\
\text { (36 weeks) }\end{array}$} & \multirow[t]{2}{*}{$\begin{array}{l}\text { Deltoid } \\
15 \text { days }\end{array}$} & \multirow[t]{2}{*}{$\begin{array}{l}\text { IHC, EM } \\
\text { (No) }\end{array}$} & \multirow{2}{*}{$\begin{array}{l}\text { Group } 1 / \text { Severe congenital } \\
\text { nemaline myopathy } \\
\text { Polyhydramnios, macrosomy } \\
\text { Severe global hypotonia, } \\
\text { respiratory distress, } \\
\text { reduced spontaneous } \\
\text { movements, ptosis, } \\
\text { arthrogryposis, hypetrichosis, } \\
\text { and macrocephaly. }\end{array}$} & \multirow[t]{2}{*}{$\begin{array}{l}\text { Yes From } \\
\text { birth }\end{array}$} & $\begin{array}{l}\text { ex177; c.24686_- } \\
\text { 24687del; p.Glu8229 } \\
\text { Gluf**18 }\end{array}$ & \multirow[t]{2}{*}{$\begin{array}{l}\text { both mutations } \\
\text { truncating/degrading }\end{array}$} & \multirow[t]{2}{*}{$\begin{array}{l}\text { Pelin et al., } \\
\text { [4] } \\
\text { Lehtokari et al., } \\
{[17]}\end{array}$} \\
\hline & & & & & & & $\begin{array}{l}\text { ex163; c.23420 } \\
23421 \text { del; p.Arg7807 } \\
\text { Serfs*16 }\end{array}$ & & \\
\hline $\begin{array}{l}\mathrm{P6}, \mathrm{M} \text {, } \\
\text { deceased } \\
\text { at } 5 \text { months }\end{array}$ & $\begin{array}{l}\text { French } \\
\text { Caucasian, } \\
\text { No }\end{array}$ & $\begin{array}{l}\text { Birth } \\
\text { (39 weeks) }\end{array}$ & $\begin{array}{l}\text { Deltoid } \\
9 \text { weeks }\end{array}$ & $\begin{array}{l}\text { IHC, IF, } \\
\text { EM (No) }\end{array}$ & $\begin{array}{l}\text { Group 2/Intermediate } \\
\text { congenital nemaline } \\
\text { myopathy Hypotonia } \\
\text { and poor spontaneous }\end{array}$ & $\begin{array}{l}\text { Yes From } \\
1 \text { month }\end{array}$ & $\begin{array}{l}\text { ex6; c.300dup; } \\
\text { p.Tyr101fs*5 int49, } \\
\text { c.6496-G > A, } \\
\text { p.2166_2234del }\end{array}$ & $\begin{array}{l}\text { exon 6: truncating/ } \\
\text { degrading exon } 50 \\
\text { skiping by RT and } \\
\text { cDNA sequencing }\end{array}$ & Present paper \\
\hline
\end{tabular}


Table 1 Clinical, laboratory, and genetic features of patients (Continued)

\begin{tabular}{|c|c|c|c|c|c|c|c|c|c|}
\hline & & & & & $\begin{array}{l}\text { movements at birth. } \\
\text { At one month } \\
\text { respiratory distress } \\
\text { and deglutition } \\
\text { problems. Elongated } \\
\text { face. High-arched } \\
\text { palate. Low-set ears. } \\
\text { Facial diplegia. }\end{array}$ & & & & \\
\hline $\begin{array}{l}\text { P7, F, } \\
\text { deceased at } \\
2 \text { and half yrs }\end{array}$ & African, Yes & $\begin{array}{l}11 \text { days } \\
\text { (39 weeks) }\end{array}$ & $\begin{array}{l}\text { Vastus } \\
\text { lateralis } \\
6 \text { months }\end{array}$ & $\begin{array}{l}\text { IHC, IF, } \\
\text { EM (Yes) }\end{array}$ & $\begin{array}{l}\text { Group } 2 / \text { Intermediate } \\
\text { congenital nemaline myopathy. } \\
\text { Apparently normal at birth. } \\
\text { Successively hypotonia and } \\
\text { deglutition problems. At } \\
1.5 \text { months development of } \\
\text { progressive respiratory failure } \\
\text { followed by recuperated } \\
\text { cardiac arrest. }\end{array}$ & $\begin{array}{l}\text { Yes From } \\
2 \text { months }\end{array}$ & $\begin{array}{l}\text { homozygous 177; } \\
\text { c.24735-24736DelA_ } \\
\text { fsx1 is this c.24735_- } \\
24736 \text { del (AG) } \\
\text { p.Arg8245fs*1 }\end{array}$ & truncating/degrading & Present paper \\
\hline \multirow[t]{2}{*}{$\begin{array}{l}\mathrm{P} 8, \mathrm{M} \\
5 \mathrm{yrs}\end{array}$} & \multirow[t]{2}{*}{$\begin{array}{l}\text { Argentinian, } \\
\text { No }\end{array}$} & \multirow[t]{2}{*}{$\begin{array}{l}6 \text { months } \\
\text { (40 weeks) }\end{array}$} & \multirow{2}{*}{$\begin{array}{l}\text { Vastus } \\
\text { lateralis } \\
6 \text { months }\end{array}$} & \multirow[t]{2}{*}{$\begin{array}{l}\text { IHC, } \\
\text { EM (Yes) }\end{array}$} & \multirow[b]{2}{*}{$\begin{array}{l}\text { Group 2/Intermediate } \\
\text { congenital nemaline } \\
\text { myopathy Hypotonia, } \\
\text { motor delay. High-arched } \\
\text { palate. Proximal and } \\
\text { distal muscle weakness. } \\
\text { Retractions of fingers. } \\
\text { Mild hyperlaxity. At } \\
1 \text { year development } \\
\text { of progressive respiratory } \\
\text { involvement necessitating } \\
\text { tracheostomy. }\end{array}$} & \multirow[t]{2}{*}{$\begin{array}{l}\text { Yes From } \\
1 \text { month }\end{array}$} & $\begin{array}{l}\text { ex139; c.20928G > } \\
\text { T; p.Gly6976Gly }\end{array}$ & \multirow{2}{*}{$\begin{array}{l}\text { RTPCR and Sanger } \\
\text { sequencing of CDNA } \\
\text { showed that instead } \\
\text { of } 105 \text { nt, exon } 139 \\
\text { contains only } 34 \text { nt: } \\
\text { frameshift and a } \\
\text { premature stop } \\
\text { codon. Exon 172; } \\
\text { truncating/degrading }\end{array}$} & \multirow[t]{2}{*}{ Present paper } \\
\hline & & & & & & & $\begin{array}{l}\text { ex172; c.24269del } \\
\text { p.Arg8090fs*54 }\end{array}$ & & \\
\hline $\begin{array}{l}P 9, M \\
11 \text { yrs }\end{array}$ & $\begin{array}{l}\text { French } \\
\text { Caucasian, } \\
\text { Yes }\end{array}$ & $\begin{array}{l}\text { Birth } \\
\text { (39 weeks) }\end{array}$ & $\begin{array}{l}\text { Deltoid } \\
10 \text { months }\end{array}$ & $\begin{array}{l}\text { IHC, IF, } \\
\text { EM (Yes) }\end{array}$ & $\begin{array}{l}\text { Group 2/Intermediate } \\
\text { congenital nemaline } \\
\text { myopathy Shortly } \\
\text { after birth severe } \\
\text { respiratory failure. } \\
\text { Tracheostomy and } \\
\text { gastrostomy at five } \\
\text { months. Facial diplegia, } \\
\text { drooling, deglutition } \\
\text { problems. Axial } \\
\text { hypotonia and } \\
\text { weakness of all limb } \\
\text { muscles. }\end{array}$ & $\begin{array}{l}\text { Yes From } \\
5 \text { months }\end{array}$ & $\begin{array}{l}\text { homozygous ex174; } \\
\text { c.24440_24441insGTCA, } \\
\text { p.Pro8148Serfs*15 }\end{array}$ & truncating/degrading & Present paper \\
\hline $\begin{array}{l}\text { P10, M, } \\
17 \text { yrs }\end{array}$ & $\begin{array}{l}\text { French } \\
\text { Caucasian, } \\
\text { No }\end{array}$ & $\begin{array}{l}6 \text { years } \\
\text { (At term) }\end{array}$ & $\begin{array}{l}\text { Deltoid } \\
6 \text { years }\end{array}$ & $\begin{array}{l}\text { IHC, IF, } \\
\text { EM (Yes) }\end{array}$ & $\begin{array}{l}\text { Group 3/Typical } \\
\text { congenital nemaline } \\
\text { myopathy. Global } \\
\text { hypotonia, deglutition } \\
\text { problems. Proximal } \\
\text { muscle weakness. } \\
\text { Facial diplegia. Nasal } \\
\text { voice. Mild respiratory }\end{array}$ & No & $\begin{array}{l}\text { int43; c.5343+5G > A } \\
\text { p.Arg1747_Thr1778del } \\
\text { ex153; c.222273del } \\
\text { p.Val7425Serfs49* }\end{array}$ & $\begin{array}{l}\text { intron 43: a splice } \\
\text { site mutation exon153: } \\
\text { truncating/degrading }\end{array}$ & Present paper \\
\hline
\end{tabular}


Table 1 Clinical, laboratory, and genetic features of patients (Continued)

\begin{tabular}{|c|c|c|c|c|c|c|c|c|c|}
\hline & & & & & $\begin{array}{l}\text { insufficiency treated } \\
\text { with discontinuous } \\
\text { non-invasive ventilation. }\end{array}$ & & & & \\
\hline $\begin{array}{l}P 11, M, \\
19 \text { years }\end{array}$ & $\begin{array}{l}\text { French } \\
\text { Antillean } \\
\text { No }\end{array}$ & $\begin{array}{l}1 \mathrm{yrs} \\
\text { (At term) }\end{array}$ & $\begin{array}{l}\text { Vastus } \\
\text { lateralis } \\
6 \text { yrs }\end{array}$ & $\begin{array}{l}\text { IHC, IF, } \\
\text { EM (Yes) }\end{array}$ & $\begin{array}{l}\text { Group } 3 / \text { Typical congenital } \\
\text { nemaline myopathy. } \\
\text { Hypotonia and feeding } \\
\text { difficulties. Delayed } \\
\text { motor milestones. Facial } \\
\text { weakness with open } \\
\text { mouth. Axial and limb } \\
\text { girdle proximal weakness. } \\
\text { Mild respiratory involvement } \\
\text { treated with discontinuous } \\
\text { non-invasive ventilation. }\end{array}$ & No & $\begin{array}{l}\text { ex175; c.24579G > A, } \\
\text { p.Ser8193Ser ex119; } \\
\text { c.18676C > T, p.Gln6226* }\end{array}$ & $\begin{array}{l}\text { ex175: a splice site } \\
\text { mutation ex119: a } \\
\text { nonsense mutation } \\
\text { (truncating/degrading) }\end{array}$ & Present paper \\
\hline $\begin{array}{l}\mathrm{P} 12, \mathrm{M}, \\
20 \mathrm{yrs}\end{array}$ & $\begin{array}{l}\text { French } \\
\text { Caucasian, } \\
\text { No }\end{array}$ & $\begin{array}{l}2-3 \text { years } \\
\text { (At term) }\end{array}$ & $\begin{array}{l}\text { Vastus } \\
\text { lateralis } \\
6 \text { years }\end{array}$ & $\begin{array}{l}\text { IHC, } \\
\text { EM (No) }\end{array}$ & $\begin{array}{l}\text { Group 3/Typical congenital } \\
\text { nemaline myopathy. } \\
\text { Difficulties in running } \\
\text { and rising stairs. Facial } \\
\text { weakness with open } \\
\text { mouth. Mild upper } \\
\text { and lower limb girdle } \\
\text { weakness. }\end{array}$ & No & $\begin{array}{l}\text { int155; c.22591-3C > } \\
\text { G; p.7531Val_- } \\
\text { Ser7564del; ex148; } \\
\text { c.21796_21810delinsT; } \\
\text { p.Pro7266fs*30 }\end{array}$ & $\begin{array}{l}\text { intron 155: a splice site } \\
\text { mutation exon 148: } \\
\text { truncating/degrading }\end{array}$ & Present paper \\
\hline$P 13, F, 52$ yrs & $\begin{array}{l}\text { French } \\
\text { Caucasian, } \\
\text { No }\end{array}$ & $\begin{array}{l}6 \text { yrs } \\
\text { (At term) }\end{array}$ & $\begin{array}{l}\text { Deltoid } \\
18 \text { years }\end{array}$ & $\begin{array}{l}\text { IHC, } \\
\text { EM (No) }\end{array}$ & $\begin{array}{l}\text { Group 3/Mild congenital } \\
\text { nemaline myopathy. } \\
\text { Difficulties in sport } \\
\text { activities in school. } \\
\text { Bilateral pes cavus. } \\
\text { Presence of mild } \\
\text { upper girdle musle } \\
\text { weakness. Diffuse } \\
\text { muscle pain. }\end{array}$ & No & $\begin{array}{l}\text { ex69; c.10043_ } \\
\text { 10046del, } \\
\text { p.Val3348Alafs } \\
\text { *43 ex49; c.6388G > C } \\
\text { p.Ala2130Pro }\end{array}$ & $\begin{array}{l}\text { ex69: truncating/ } \\
\text { degrading ex49: } \\
\text { missense on the } \\
\text { acting binding site }\end{array}$ & Present paper \\
\hline P14, F, 37 yrs & $\begin{array}{l}\text { French } \\
\text { Caucasian, } \\
\text { No }\end{array}$ & $\begin{array}{l}2 \text { yrs } \\
\text { (At term) }\end{array}$ & $\begin{array}{l}\text { Deltoid } \\
21 \text { yrs }\end{array}$ & $\begin{array}{l}\text { IHC, IF, } \\
\text { EM (Yes) }\end{array}$ & $\begin{array}{l}\text { Group 3/Mild congenital } \\
\text { nemaline myopathy. } \\
\text { Frequent falls. Difficulties } \\
\text { in running, rising stairs. } \\
\text { Jaw contractures. Nasal } \\
\text { voice. Elongated face. } \\
\text { Respiratory involvement. } \\
\text { Axial weakness with } \\
\text { difficulties in neck flexion. } \\
\text { Asymmentrical distal } \\
\text { weaakness with foot } \\
\text { drop (right > left). } \\
\text { Proximo-distal weakness. }\end{array}$ & No & $\begin{array}{l}\text { int17; c.1569+1G > } \\
\text { A p.His491_Asp523del } \\
\text { ex176; c.24606del } \\
\text { p.Ala8203Glnfs*13 }\end{array}$ & $\begin{array}{l}\text { intron 17: a splice } \\
\text { site mutation } \\
\text { exon 176: truncating/ } \\
\text { degrading }\end{array}$ & Present paper \\
\hline
\end{tabular}


conjugated secondary antibodies for one hour (Alexa Fluor-488 green goat anti-rabbit antibody, and Alexa Fluor-594 red goat anti-mouse antibody, Molecular Probes, Cergy Pontoise, France). A set of control slides was prepared with omission of the primary antibodies.

\section{Electron microscopy}

Detailed electron microscopy analysis was prospectively performed in thirteen patients. Small muscle specimens were fixed with glutaraldehyde (2.5\%, $\mathrm{pH} 7.4)$, post fixed with osmium tetroxide (2\%), dehydrated and embedded in resin (EMBed-812, Electron Microscopy Sciences, USA). Ultra-thin sections from at least three small blocks from each patient were stained with uranyl acetate and lead citrate. The grids were observed using a Philips CM120 electron microscope $(80 \mathrm{kV}$; Philips Electronics NV, Eindhoven, The Netherlands) and were photo documented using a Morada camera (Soft Imaging System, France).

\section{Muscle contractility experiments}

To investigate whether the contractile performance is affected in muscle biopsies from patients with mutations in the nebulin gene, we performed skinned muscle fibre contractility experiments. Small strips were dissected from muscle biopsies of patients P1 (Group 1), P7, P8 and P9 (Group 2), and P10, P11 and P14 (Group 3) and were skinned overnight as described previously [32]. The skinning procedure renders the membranous structures in the muscle fibres permeable, which enables activation of the myofilaments with exogenous $\mathrm{Ca}^{2+}$. Preparations were washed thoroughly with relaxing solution and stored in $50 \%$ glycerol/relaxing solution at $-20^{\circ} \mathrm{C}$. Small muscle preparations (cross-sectional area $\sim 0.002 \mathrm{~mm}^{2}$ ) were dissected from the skinned strips, and were mounted using aluminum T-clips between a length motor (ASI 403A, Aurora Scientific Inc., Ontario, Canada) and a force transducer element (ASI 315C-I, Aurora Scientific Inc., Ontario, Canada) in a single fibre apparatus (ASI 802D, Aurora Scientific Inc., Ontario, Canada) that was mounted on an inverted microscope (Zeiss Axio Observer A1). Sarcomere length was set using a high speed VSL camera and ASI 900B software (Aurora Scientific Inc., Ontario, Canada). Mechanical experiments were performed at a sarcomere length of $\sim 2.2 \mu \mathrm{m}$, a length selected to minimize force differences due to shorter thin filaments in fibres from nemaline myopathy patients with nebulin mutations [32]. Fibre width and diameter were measured at three points along the fibre and the cross-sectional area was determined assuming an elliptical cross-section. Two different types of bathing solutions were used during the experimental protocols: a relaxing solution (40 mM BES; $10 \mathrm{mM}$ EGTA; $6.86 \mathrm{mM} \mathrm{MgCl}_{2}$; $5.96 \mathrm{mM}$ Na-ATP; $3.28 \mathrm{mM}$ K-propionate; $33 \mathrm{mM}$ creatine phosphate; $1 \mathrm{mM}$
DTT; 0.5 mM PMSF; 0.2 mM Leupeptin; 0.05 mM E64) and an activating solution (40 $\mathrm{mM}$ BES; $10 \mathrm{mM}$ $\mathrm{CaCO}_{3}$-EGTA; $6.64 \mathrm{mM} \mathrm{MgCl}_{2} ; 6.23 \mathrm{mM} \mathrm{Na-ATP}$; $2.1 \mathrm{mM}$ K-propionate; $15 \mathrm{mM}$ creatine phosphate; $1 \mathrm{mM}$ DTT; 0.5 mM PMSF; $0.2 \mathrm{mM}$ Leupeptin; $0.05 \mathrm{mM}$ E64). The temperature of the bathing solutions was controlled by a TEC controller (ASI 825A, Aurora Scientific Inc. Ontario, Canada). Muscle preparations were mounted in a relaxation solution at $1^{\circ} \mathrm{C}$. Subsequently, the muscle preparations were pre-activated by switching to an activation solution at $1^{\circ} \mathrm{C}$. In that way the myofibres are loaded with calcium, but no force is generated. By rapid switching to an activation solution at $20^{\circ} \mathrm{C}$, the fibres were activated and force was generated. When the force trace reached a plateau, the muscle fibres were slacked to $70 \%$ of their original length followed by a rapid restretch to the original length after 30 milliseconds. This procedure allows the force to redevelop from zero [33]. The rate of tension redevelopment was calculated by fitting a bi-exponentional through the force redevelopment curve. The first-order rate constant $k_{1}$ reflects crossbridge cycling kinetics and was therefore used in the analyses [34].

\section{Myosin heavy chain composition of bundles used for contractility experiments}

For determination of the myosin heavy chain isoform composition of the muscle fibre preparations we used specialized SDS-PAGE [32]. In brief, muscles fibres were denatured by boiling for 2 minutes in SDS sample buffer. The stacking gel contained a $4 \%$ acrylamide concentration (pH 6.7), and the separating gel contained 7\% acrylamide $(\mathrm{pH} 8.7)$ with $30 \%$ glycerol $(\mathrm{v} / \mathrm{v})$. The gels were run for $24 \mathrm{~h}$ at $15^{\circ} \mathrm{C}$ and a constant voltage of $275 \mathrm{~V}$. Lastly, the gels were silver-stained, scanned, and analysed with One-D scan EX software (Scanalytics Inc., Rockville, MD, USA).

The ethical committee of La Pitié-Salpêtrière Hospital (CCPPRB) approved this study.

\section{Results \\ Clinical findings}

Five patients were female and 9 were male. Clinical summary, laboratory features, a complete list of morphological methods, functional studies applied to muscle biopsies, and genetic characterization of all patients is provided in Table 1. Patient 6 and 7 were classified to be a part of intermediate congenital nemaline myopathy group because they were breathing and moving at birth. However, these patients developed soon after birth a severe clinical picture and they were never able to achieve respiratory independence and/or ambulation. They eventually deceased at 5 months, and 32 months respectively. They therefore present a phenotype in between a severe and an intermediate congenital nemaline myopathy. 


\section{Molecular data}

To identify the genetic cause in this cohort of patients, we performed exome enrichment and sequencing on genomic DNA from the patients and their parents. Exome sequencing allows a rapid and parallel screening of most human genes, and is suitable and efficient for the diagnosis of neuromuscular diseases and the analysis of large genes such as $N E B$, frequently mutated in NM [15]. This approach also covers any newly discovered gene for the disorder. For all patients presented here we found known or novel variations in the $N E B$ gene. These changes were confirmed by Sanger sequencing, and their familial segregation validated when parent DNA available. In all the patients, at least one of the two compound heterozygous mutations was a truncating mutation (frameshift or nonsense mutation) leading to protein truncation or degradation, or both (Table 1). The second mutations were, either a frameshift mutation, deletion of several amino acids in frame (splice site mutations), a non-conservative missense change close to an actin binding site (P13), or synonymous variants in P3, P8, and P11 that all impacted on splicing. Most of the mutations (18) affected ubiquitously expressed exons, while six were in the alternatively spliced exons and one in an exon of the triplicated region of eight exons. Overall, most mutations were predicted to lead to a truncated or absent protein. There was no obvious correlation between the type of mutation or its location on the protein and the clinical severity.

\section{Morphological findings Histological and histochemical features Group 1}

A similar morphological pattern characterised by marked fibre size variability was noted in all biopsies from G1. We constantly identified two populations of fibres; the first characterized by muscle fibres of predictably normal or slightly augmented size, and the second one consisting of severely atrophic fibres (Figure 1A; arrows). Small rounded/ globular inclusions staining red by the $\mathrm{mGT}$, corresponding to nemaline bodies, were present in less than half of muscle fibres (Figure 1A). Nemaline bodies occupied both normal-sized and atrophic fibres (Figure 1A). They presented a dispersed or, more often, a subsarcolemmal distribution (Figure 1A). Some atrophic fibres appeared completely occupied by nemaline bodies. The percentage of muscle fibres harbouring rods was to $22 \%$ to $33 \%$ (Figure 1D, Group 1, red). The oxidative enzyme reactions revealed more than $30 \%$ of fibres presenting uneven staining of the intermyofibrillar network (not shown). The latter did not always correspond to the areas occupied by nemaline bodies, suggesting the presence of some degree of sarcomeric disruption. ATPase techniques did not reveal type 1 fibre predominance in any of the patients in G1. The majority of fibres staining differently from type 1 are probably undifferentiated fibres (not shown).

\section{Group 2}

The morphological pattern found in this group was heterogeneous compared with G1 biopsies. While in P6, and P7 samples we noticed the presence of two populations of muscle fibres (predictably normal size and severely atrophic) (Figure 1B; arrows), P8, and P9 showed a mild variation of fibres size without any particular topography (not shown). Conversely to G1 nemaline bodies were present in the vast majority of fibres (mean: 95\% to 100\%; Figure 1D, Group 2, blue) and presented a variable shape varying from oval to elongated. The oxidative enzyme reactions revealed some alteration of the intermyofibrillar network, probably corresponding to rod accumulation or myofibrillar disorganization with oxidative techniques. ATPase techniques showed type 1 fibre predominance in P6, and P8. Type 1 uniformity was noted in P7 and P9.

\section{Group 3}

In this group we noticed mild variation of fibre size except in P12 where some atrophic rounded fibres were identified (not shown). Nemaline bodies presented a constant elongated shape and formed well separated clusters both in subsarcolemmal and cytoplasmic areas (Figure 1C). There was a large variability in the percentage of fibres harbouring rods. While P10, P13 and P14 presented rods in almost all fibres (97-99\%), P11 had 60\% and P12 31\% of fibres with rods, respectively (Figure 1D, Group 3, green). ATPase techniques showed almost complete type 1 uniformity in G3 muscle biopsies. The areas of muscle fibres containing rods lacked ATPase staining.

\section{Summary}

Overall, severe NM was associated with a fibre size variability, presence of rods in about $1 / 3$ of fibres, and a high percentage of undifferentiated fibres. By contrast a higher percentage of fibres with rods, and a type 1 fibre predominance/uniformity was noted in the intermediate and typical NM patients. The amount of nemaline bodies seems to be inversely related to clinical severity.

\section{Immunohistochemistry and immunofluorescence Group 1}

In P1 we identified many fibres expressing developmental, neonatal, fast and/or slow myosin. Immunofluorescence studies showed absence of type $2 \mathrm{X}$ myosin.

\section{Group 2}

In P6 we identified many fibres co-expressing slow and fast myosins. Developmental and neonatal myosins were 

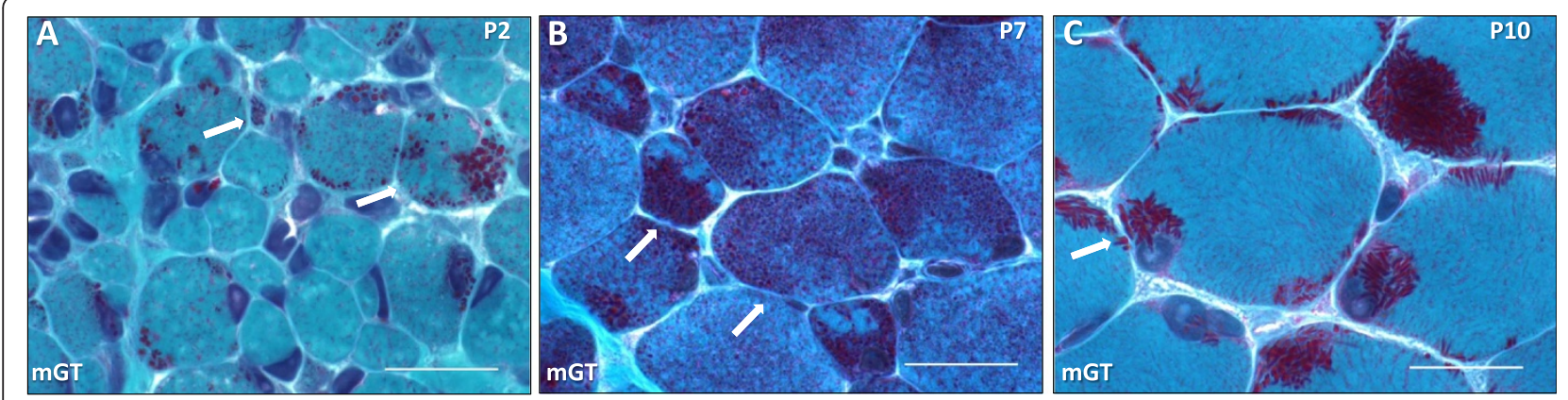

D

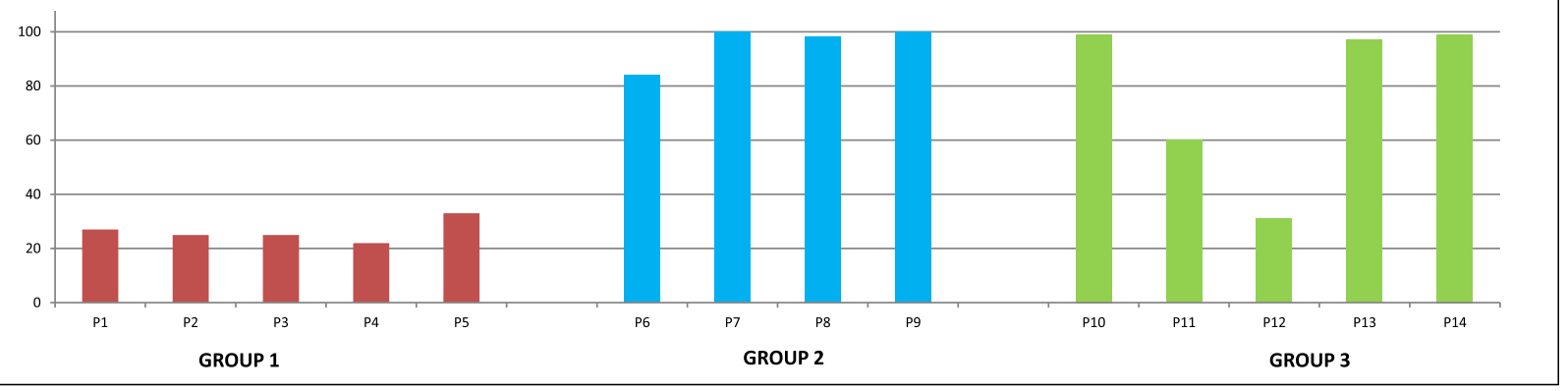

Figure 1 Light microscopy and percentage of fibers with rods. Modified Gomori trichrome stainings. A and $\mathbf{B}$. Nemaline bodies in muscle biopsies from P2 (G1) and P7 (G2) have a rounded/ovoid shape. They are present in both normal size, and atrophic fibres (indicated by arrows). C. Nemaline bodies in muscle biopsy from P11 have an elongated shape (indicated by arrows) and they are localized in subsarcolemmal and perinuclear areas. Scale bars represent $20 \mu \mathrm{m}$ for mGT stainings. D. Representation of percentage of rod occupied fibres in Group 1 (red), Group 2 (blue), and Group 3 (green). See text for explanations.

expressed in a minority of fibres. In P8 we identified occasional fibres expressing developmental myosin and less than $5 \%$ of fibres expressing neonatal myosin; there was partial co-expression of fast and slow myosins. P9 showed unique expression of slow myosin both in immunohistochemical, and immunofluorescence studies.

\section{Group 3}

P10, P11, and P14 showed slow myosin uniformity using both techniques. No fibres expressing developmental and/or neonatal myosins were noted.

\section{Electron microscopy}

\section{Group 1}

The prominent ultrastructural finding in all G1 patients was the diffuse myofibrillar dissociation. The myofibrils appeared thinner, and smaller than in age-matched controls (Figure 2A). The latter suggested either a defect in sarcomeric structure establishment either the lost of it. Remnants of sarcomeres were intermingled with organelles (e.g. mitochondria) or glycogen granules (Figure 2A). Globular/ovoid nemaline bodies were scattered or distributed in subsarcolemmal and perinuclear areas (Figure 2B, and $\mathrm{C}$ ). Some fibres were completely occupied by them (Figure 2C). At higher magnification globular/ovoid rods presented thin filaments projecting from their thinnest edges (Figure 2D).

\section{Group 2}

This group presented a milder degree of myofibrillar dissociation accompanying dispersed or clustered nemaline bodies (Figure 3A, and 3B). Rods showed the typical lattice structure resembling Z-disc material at very high magnification (Figure 3C). In P9 we noticed the presence of some typical cytoplasmic bodies with a dense core, and a clear halo of fine filaments (not shown).

\section{Group 3}

We found a homogenous picture characterised by the presence of well-separated clusters of subsarcolemmal (Figure 4A), perinuclear, and less often cytoplasmic nemaline bodies (Figure 4B). The rods were always surrounded by thin filaments and amorphous material. The sarcomeric structure was overall conserved (Figure 4A, and 4B).

\section{Summary}

Taken together, our results suggest that myofibrillar dissociation correlated with clinical severity. 

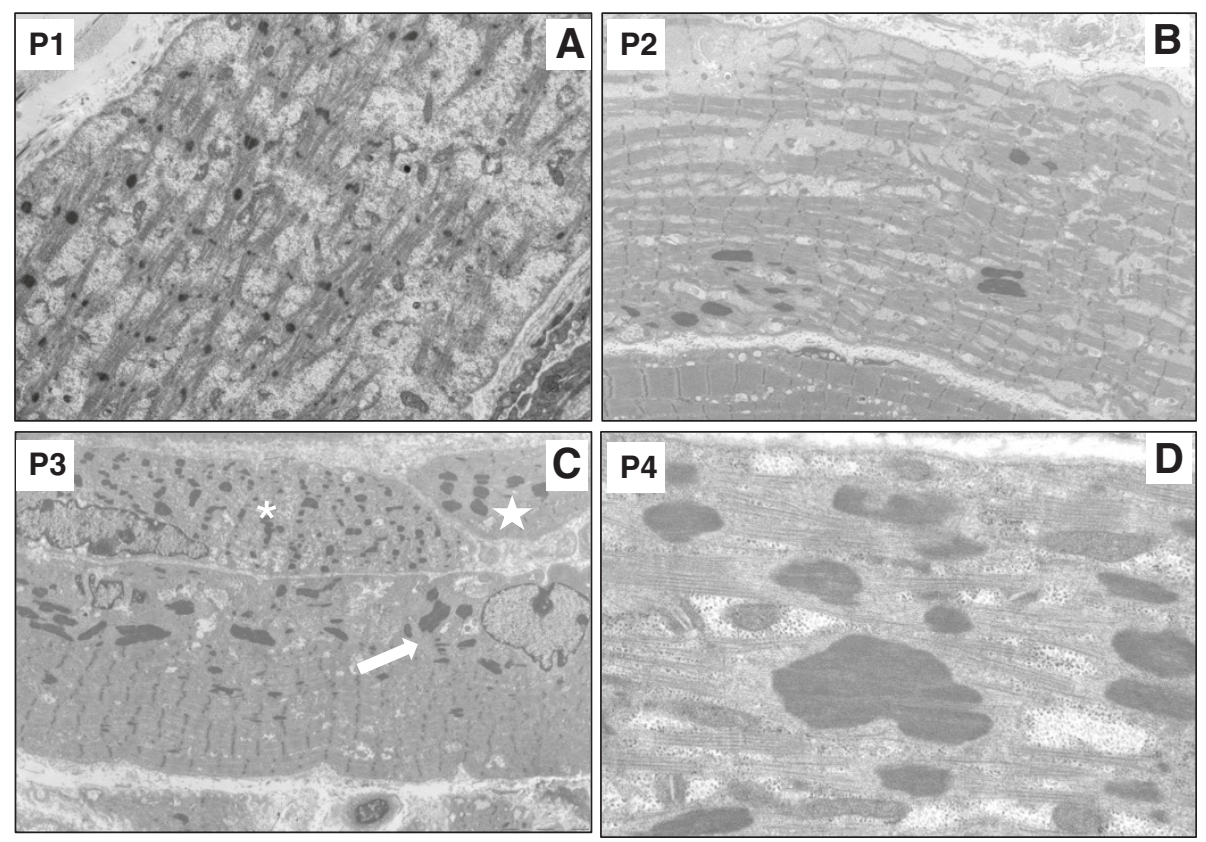

Figure 2 Electron microscopy for group G1-severe NM. A. P1. Sarcomeric structure is completely disrupted. Fragment of sarcomeres are intermingled with amorphous material containing organelles and glycogen granules. B. P2. Scattered globular ovoidal nemaline bodies are found inside a fiber showing partial sarcomeric alteration. C. P3. Presence of three muscle fibres presenting different degree of alterations. Nemaline bodies are found in subsarcolemmal areas (indicated by an arrow). The fibre above the first one shows sarcomeric disarray and thicknened Z-lines probably leading to globular rods formation (indicated by an asterisk). A small atrophic fibre is completely invaded by rounded nemaline bodies (indicated by a star). D. P4. Globular nemaline bodies present thin filaments coming out from the thinner edges. Original magnification: A. 11,000x. B. $7,000 \times$ C. $8,400 \times$ D. $51,000 \times$.

\section{Muscle contractility experiments}

The maximal force generation capacity of the muscle fibre preparations was normalized to their cross-sectional area (i.e. maximal active tension) (Figure 5). An overview of the maximal active tension and the rate of tension redevelopment of the muscle fibres are summarized in Table 2. Data are presented as mean \pm SEM.

\section{Myosin heavy chain analyses}

The myosin heavy chain (MHC) gel electrophoresis experiments revealed that muscle preparations from biopsies from patient $\mathrm{P} 1$ contained both neonatal isoforms as well as type 1 and type $2 \mathrm{~A}$ isoforms: $(9.2 \pm 2.8 \% \mathrm{MHC}$ neonatal; $40.0 \pm 3.2 \% \mathrm{MHC}$ type $1 ; 50.8 \pm 1.3 \% \mathrm{MHC}$ type 2A). Muscle preparations from biopsies of P7 and P8 (group 2) contained both MHC type 1 and MHC type 2A isoforms: P7 (66.6 $\pm 6.6 \% \mathrm{MHC}$ type 1 and $33.4 \pm 6.6 \%$ MHC type $2 \mathrm{~A})$ and P8 $(40.8 \pm 12.0 \%$ MHC type 1 and $59.2 \pm 12.0 \% \mathrm{MHC}$ type $2 \mathrm{~A})$, respectively. All other patient biopsies (P9, P10, P11 and P14) showed exclusively myosin heavy chain type 1 isoforms.

\section{Discussion}

In our tertiary Center for Neuromuscular Disorders we perform a detailed clinical, morphological, and genetic analysis of large cohorts of patients presenting NM. Due to the genetic heterogeneity of NM, and the difficulties encountered in the molecular screening of the 'giant' $N E B$ gene [15,35], in France and Finland an integrated approach combining next generation sequencing and dHPLC/Sanger sequencing was set-up. Our strategy allowed the identification of ten new families harbouring $N E B$ mutations. All patients presented autosomal recessive pattern of inheritance and either homozygous or compound heterozygous pathogenic variants. Our results confirm that $N E B$ is one of the most frequently mutated NM genes, accounting for almost half of the genetically identified NM patients screened for the known genes associated with NM [3-11].

We undertook a detailed clinical histological, and, when possible, muscle functional analysis in a cohort of fourteen $N E B$-mutated subjects whose muscle biopsy was available in our laboratory. We comment on relevant findings encountered.

NEB-mutated patients revealed a wide pathological spectrum and showed recurrent morphological pattern with some overlap among the clinical groups. Lethal/severe NM subjects (G1) presented: high degree of myofibrillar dissociation and smallness revealed by electron microscopy, scattered globular/ovoid nemaline bodies occupying one third of muscle fibres, and absence of type 1 predominance with myosins ATPases techniques (Figure 2). Intermediate 


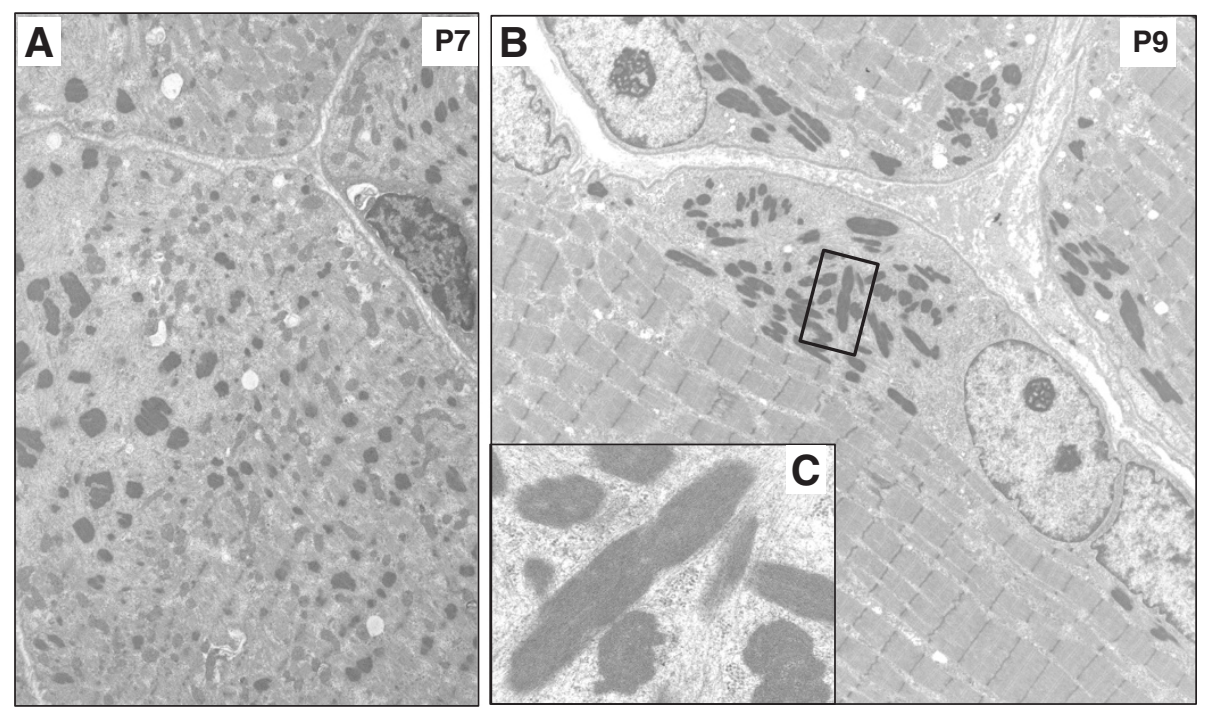

Figure 3 Electron microscopy for group G2-intermediate NM. A. P7. Globular ovoid nemaline bodies associated with partial sarcomeric disarray strongly resembling the pathologic picture of G1. B. P9. Presence of elongated nemaline bodies in perinuclear and subsarcolemmal areas. The latter are well separated from well-preserved sarcomeric structure. C. P9. Higher magnification of an elongated nemaline body. The typical periodic net structure composing rods is clearly recognisable. Thin filament spread out of the thinnest edges of rods. Sparse thin filaments are found around the rods intermingled with glycogen granules. Original magnification: A. 11,000x B. 6,400x C. 94,000x.

congenital myopathy patients (G2) showed features similar to $\mathrm{G} 1$ in $\mathrm{P} 6$ and P7, even though the presented higher percentage of rods and type 1 predominance, and well-separated clusters of rods associated with type 1 predominance or uniformity in the other patients. It is noteworthy that P6 and P7 deceased at 5 months, and 2 and a half year, respectively. For this reason these patients could be considered as a 'Longer survivor severe congenital $N M$ ' subgroup due to their 'midway' clinical and morphological features between G1 and G2. Group 3 had a preserved sarcomeric structure with clusters of elongated rods invariantly associated with type 1 predominance/uniformity.

In summary we show a large pathological spectrum ranging from severely damaged sarcomeres with scattered nemaline bodies to globally preserved muscle with clusters of well separated rods occupying the majority of myofibres. Interestingly the degree of sarcomeric disruption directly

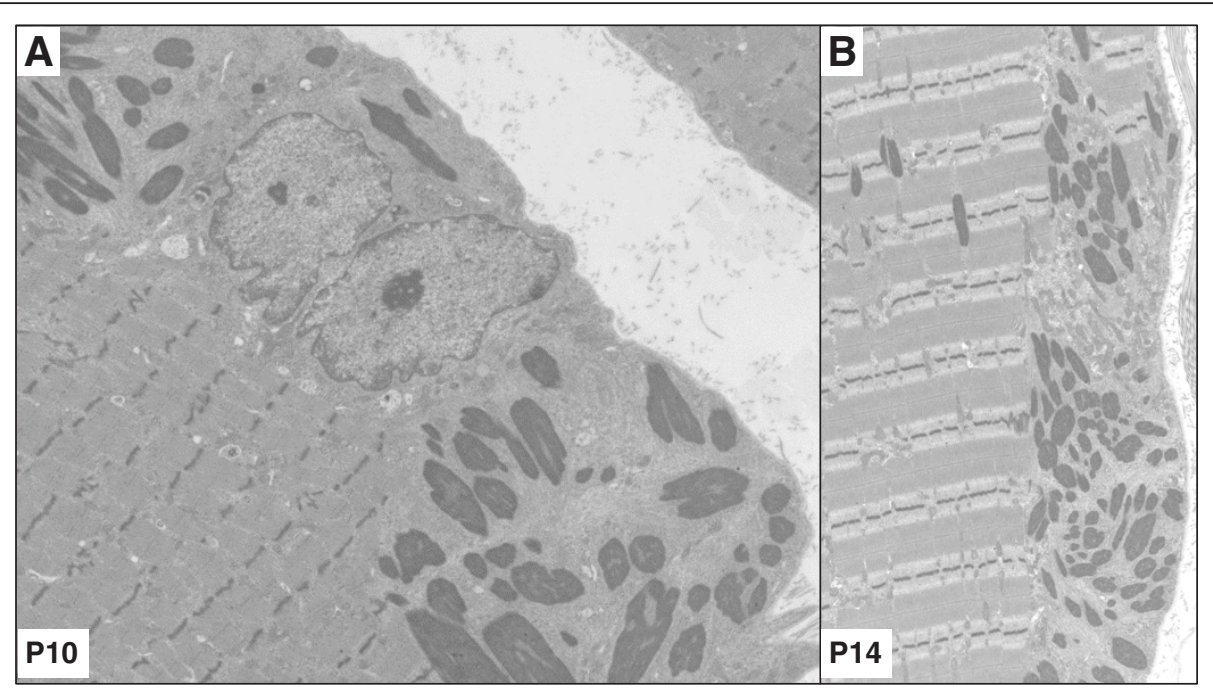

Figure 4 Electron microscopy for group G3-typical-mild NM. A. P10, G3. Presence of globally preserved sarcomeric structure associated with a cluster of elongated nemaline bodies in the subsarcolemmal areas of muscle fibres. P10. B. P14. Well-delimited clusters of rods surrounded by thin filaments. Original magnification: A. 9,000x B. 8,200x. 


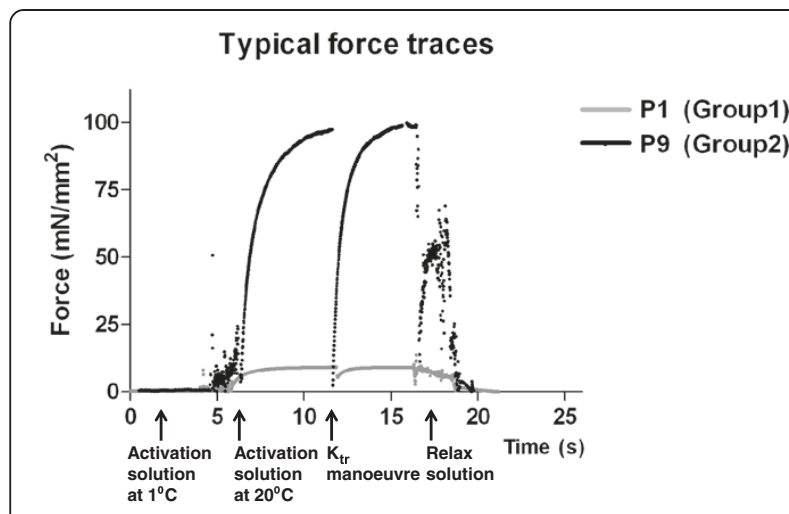

Figure 5 Typical force traces of skinned myofibers from nemaline myopathy patients with nebulin mutations. Typical examples from force traces of a skinned muscle preparation from P1 from Group1 (in grey) and P9 from Group2 (in black). Myofibres are pre-activated by exposure to an activation solution at $1^{\circ} \mathrm{C}$. By rapid switching to an activation solution at $20^{\circ} \mathrm{C}$, the fibers are activated and force is generated. When the force trace reaches a plateau, the myofibres are slacked to $70 \%$ of their original length followed by a rapid restretch to the original length after 30 milliseconds ( $K_{t r}$ manouvre). When force generation has reached a plateau again, the muscle preparation is exposed to a calcium-free solution to induce relaxation.

related to clinical severity whereas the number of rod invaded fibres seemed to be inversely correlated.

We undertook this study to search for genotypephenotype correlations. Taken together, the vast majority of $N E B$ mutations are predicted to lead to a degradation and or denaturation of many nebulin isoforms. Markedly reduced amounts of nebulin in muscle samples from patients homozygous for exon 55 deletions have been reported previously [36]. P2 in this study is homozygous for the same exon 55 deletion, resulting in an in-frame deletion of 35 amino acids, and subsequent protein degradation. Nonsense mRNA-mediated decay was demonstrated by RT-PCR in P1, who carries a heterozygous nonsense mutation in exon 45 . P1 is also heterozygous for a splice site mutation in intron 122, which was shown to cause exon 122 skipping, resulting in in-frame deletion of 35 amino acids [15]. Splice site mutations are, however, often "leaky", i.e. some transcripts are spliced correctly, whereas others are incorrectly spliced. Therefore, it seems plausible that $\mathrm{P} 1$, as well as the other patients with splice site mutations (P3, P6, P8, P10, P11, P12 and P14) express small amounts of normal nebulin in their muscles. Markedly reduced amounts of nebulin in muscle have been reported in one patient compound heterozygous for a splice site mutation and a frameshift mutation in constitutively expressed exons [37]. The mutations in alternatively spliced exons (exons 174, 175, 176 and 177) only affect nebulin isoforms expressing these exons, leaving other isoforms unaffected. Consequently reduced amounts of nebulin and absence of some isoforms, precluding an appropriate thin filaments assembly, might be responsible for the drastic myofibrillar dissociation revealed in G1 [Figure 4]. Functional studies confirmed that G1 patients' muscle fibers generate very low force. Although one could argue that age confounded our findings, previous work from our group revealed no major differences in the contractile performance of myofibres isolated from young (age 2-5 years) versus adult (age 20-30) human control biopsies [37]. Note that G1 patients' muscle fibres expressed only very low levels of neonatal myosin heavy chain isoforms; such low levels are unlikely to account for the major loss of force in this patient. We therefore suggest that nebulin degradation/absence translates into sarcomeric disarray and/or altered actomyosin interaction. This could be responsible for low force generation producing global hypotonia, muscle akinesia, and arthrogryposis. Concordantly, a recent study reported that low levels of nebulin in skeletal muscle are probably responsible for the foetal akinesia and arthrogryposis sequence phenotype [36].

How nebulin deficiency results in nemaline bodies formation is yet to be understood. Some authors suggested that a truncated nebulin would disrupt myofibrillar connectivity leading to Z-disc displacement and, eventually, rods formation [38]. Analysis of nemaline bodies features in our cohort revealed differences in shape across the three groups. These tended to be globular/ovoid in G1, elongated in the other groups. The specificity of this finding is uncertain. What is more striking is that in P8, and P9 from G2 and in all G3 patients, the majority of fibres harboured rod clusters confined to subsarcolemmal and/or perinuclear areas. The myofibrillar structure surrounding them was overall preserved. Some unknown process could try to circumscribe the protein aggregates and avoid a perturbation of muscle contraction. This could explain why muscle fibers in G2 and G3 patients showed a better contractile performance, probably translating into a milder clinical phenotype. It is tempting to speculate that mutations encountered in these groups affect only specific nebulin isoforms, which is certainly true for patients P7, P8, P9, P11, and P14 who all have at least one mutation in an alternatively spliced exon. In this scenario, residual normal isoforms could allow a proper thin filament

Table 2 Muscle contractility data of permeabilized fibers from nemaline myopathy patients with NEB mutations

\begin{tabular}{llllllll}
\hline & $\mathbf{P 1}$ & $\mathbf{P 7}$ & $\mathbf{P 8}$ & $\mathbf{P 9}$ & $\mathbf{P 1 0}$ & $\mathbf{P 1 1}$ & P14 \\
\hline Maximal active tension $\left(\mathbf{m N} / \mathbf{m m}^{\mathbf{2}}\right)$ & $8.1 \pm 1.5$ & $18.8 \pm 2.7$ & $76.9 \pm 10.0$ & $90.9 \pm 10.5$ & $55.5 \pm 7.1$ & $102.2 \pm 21.2$ & $61.4 \pm 12.2$ \\
Rate of tension redevelopment $\left(\mathbf{k}_{\mathbf{1}}\left(\mathbf{s}^{-\mathbf{1}}\right)\right)$ & $5.1 \pm 0.4$ & $4.5 \pm 0.7$ & $5.0 \pm 0.5$ & $3.4 \pm 0.2$ & $3.6 \pm 0.2$ & $4.0 \pm 0.6$ & $3.8 \pm 0.5$ \\
\hline
\end{tabular}


assembly, while the altered ones might be responsible for protein aggregation/rod formation. If this turned to be true, the presence of rods might be considered unrelated to muscle contractility disturbances. Following this reasoning we could imagine that an additive effect of degraded nebulin isoforms would determine clinical severity.

Type 1 predominance or type uniformity has been reported as a very common feature associated with NM, and many other structural congenital myopathies [39]. It is speculated that this is due to a disturbance of fibre differentiation before phase three of muscle differentiation (35th weeks) [40]. A severe NEB mutated family composed by two brothers has been reported as not having type 1 fibre predominance. However, biopsied muscle and age at muscle biopsy were not specified [41]. In the present study we performed type fibre distribution analysis on ATPase techniques. Surprisingly, all G1 patients failed to show type 1 predominance and constantly showed absence of $2 \mathrm{~B}$ fibers. Immunostainings for different myosins isoforms (foetal neonatal, fast and slow) revealed a certain degree of myosin isoforms co-expression in numerous fibres suggesting the presence of undifferentiated fibers. This contrasted with the other groups where type 1 predominance/uniformity was present. This finding suggests that G1 $N E B$-mutated patients were not able to switch towards type 1 predominance due to a possible alteration of muscle maturation. It is tempting to speculate that aberrations in fibre-typing, and absence of type $2 \mathrm{~B}$ seen with ATPase techniques are due to changes in isoforms imbalance more than related to age at muscle biopsy. Additionally the presence of high percentages of undifferentiated fibres encountered in G1 might turn out to be an important prognostic factor. In fact muscle biopsy analysis of new-borns presenting arthrogryposis and a pathological picture characterised by sarcomeric dissociation, scattered nemaline bodies absence of type 1 predominance and type 2B fibres with ATPases techniques might orientate toward $N E B$ mutations. This is something distinctive from other severe form of congenital myopathies commonly showing type 1 predominance as a prominent feature [42]. In particular we recently demonstrated that MTM1-mutated boys presenting an extremely severe clinical phenotype all had type 1 predominance in their biopsies, regardless of the biopsied muscle, and the gestational age [28].

In conclusion, this study adds on the clinical, morphological and functional characterization of the most recurrent form of NM. We assessed morphological and functional heterogeneity in NEB-mutated NM patients and identified a correlation between disease severity on the one hand, and ultrastructural myofibrillar abnormalities and contractility on the other. We suggest that myofibrillar dissociation and smallness is a primary defect causing the disease while nemaline bodies could be due to a collateral mechanism.
Competing interests

The authors declare that they have no competing interests.

\section{Acknowledgements}

We thank M. Beuvin G. Brochier, E. Lacène, and L. Manéré, (Institut de Myologie, Unité de Morphologie Neuromusculaire), and Dr Maria Saccoliti (Instituto de Investigationes Neurologicas FLENI) for their excellent technical help.

\section{Funding}

This work was supported in part by the Assistance Publique-Hôpitaux de Paris (AP-HP), the Institut National de la Santé et de la Recherche Médicale (INSERM), the Association Française contre les Myopathies (AFM), the Association Institut de Myologie (AIM), the Agence Nationale de la Recherche (ANR-11-BSV1-026), the Sigrid Jusélius Foundation, the Finska Läkaresällskapet and the Medicinska understödsföreningen Liv och Hälsa r.f.

\section{Author details}

${ }^{1}$ Unité de Morphologie Neuromusculaire, Institut de Myologie, Groupe Hospitalier Universitaire La Pitié-Salpêtrière, Paris, France. ${ }^{2}$ Department of Neurological, Neurosurgical, and Behavioral Sciences, University of Siena, Siena, Italy. ${ }^{3}$ Inserm, U974, Paris F-75013, France. ${ }^{4}$ Université Pierre et Marie Curie- Paris 6, UM 76, Inserm, U974, CNRS, UMR 7215, Institut de Myologie, Paris F-75013, France. ${ }^{5}$ Centre de référence de Pathologie Neuromusculaire Paris-Est, Institut de Myologie, GHU La Pitié-Salpêtrière, Assistance

Publique-Hôpitaux de Paris, Paris, France. ${ }^{6}$ Department of Medical Genetics, Haartman Institute, University of Helsinki, and the Folkhälsan Institute of Genetics, Biomedicum Helsinki, Helsinki, Finland. ${ }^{7}$ Department of Translational Medicine, IGBMC, INSERM U964, UMR7104, Strasbourg University, Illkirch, France. ${ }^{8}$ Department of Physiology, VU University medical center, Amsterdam, the Netherlands. ${ }^{9}$ AP-HP, Service de Pédiatrie, Hôpital Raymond Poincaré, Garches; Hôpitaux, Universitaires Paris-lle-de-France Ouest, Pôle pédiatrique; Centre de Référence, Maladies Neuromusculaires Garches-Necker-Mondor-Hendaye (GNMH), Université Versailles Saint-Quentin en Yvelines (UVSQ), Garches, France. ${ }^{10}$ Hospital Nacional de Pediatría J.P. Garrahan, and Instituto de Investigaciones Neurologicas, FLENI, Buenos Aires, Argentina. ${ }^{11} \mathrm{CHU}$ de Fort de France, Martinique, France. ${ }^{12} \mathrm{BGl}$-Shenzhen, Shenzhen 518083, China. ${ }^{13}$ Department of Biosciences, Division of Genetics, University of Helsinki, Helsinki, Finland.

Received: 18 March 2014 Accepted: 20 March 2014

Published: 12 April 2014

\section{References}

1. Romero NB, Sandaradura SA, Clarke NF (2013) Recent advances in nemaline myopathy. Curr Opin Neurol 26(5):519-526

2. Wallgren-Pettersson C, Laing NG (2001) Report of the 83rd ENMC International Workshop: 4th Workshop on Nemaline Myopathy, 22-24 September 2000, Naarden, The Netherlands. Neuromuscul Disord 11(6-7):589-595

3. Nowak KJ, Wattanasirichaigoon D, Goebel HH, Wilce M, Pelin K, Donner K, Jacob RL, Hübner C, Oexle K, Anderson JR, Verity CM, North KN, lannaccone ST, Müller CR, Nürnberg P, Muntoni F, Sewry C, Hughes I, Sutphen R, Lacson AG, Swoboda K, Vigneron J, Wallgren-Pettersson C, Beggs AH, Laing NG (1999) Mutations in the skeletal muscle alpha-actin gene in patients with actin myopathy and nemaline myopathy. Nat Genet 23(2):208-212

4. Pelin K, Hilpelä P, Donner K, Sewry C, Akkari PA, Wilton SD, Wattanasirichaigoon D, Bang ML, Centner T, Hanefeld F, Odent S, Fardeau M, Urtizberea JA, Muntoni F, Dubowitz V, Beggs AH, Laing NG, Labeit S, de la Chapelle A, Wallgren-Pettersson C (1999) Mutations in the nebulin gene associated with autosomal recessive nemaline myopathy. Proc Natl Acad Sci U S A 96(5):2305-2310

5. Donner K, Ollikainen M, Ridanpää M, Christen HJ, Goebel HH, de Visser M, Pelin K, Wallgren-Pettersson C (2002) Mutations in the beta-tropomyosin (TPM2) gene-a rare cause of nemaline myopathy. Neuromuscul Disord 12(2):151-158

6. Laing NG, Wilton SD, Akkari PA, Dorosz S, Boundy K, Kneebone C, Blumbergs P, White S, Watkins H, Love DR, Love DR (1995) A mutation in the alpha tropomyosin gene TPM3 associated with autosomal dominant nemaline myopathy. Nat Genet 9(1):75-79 
7. Johnston JJ, Kelley Rl, Crawford TO, Morton DH, Agarwala R, Koch T, Schäffer AA, Francomano CA, Biesecker LG (2000) A novel nemaline myopathy in the Amish caused by a mutation in troponin T1. Am J Hum Genet 67(4):814-821. Epub 2000 Aug 21

8. Agrawal PB, Greenleaf RS, Tomczak KK, Lehtokari VL, Wallgren-Pettersson C, Wallefeld W, Laing NG, Darras BT, Maciver SK, Dormitzer PR, Beggs AH (2007) Nemaline myopathy with minicores caused by mutation of the CFL2 gene encoding the skeletal muscle actin-binding protein, cofilin-2. Am J Hum Genet 80(1):162-167. Epub 2006 Nov 14

9. Sambuughin N, Yau KS, Olivé M, Duff RM, Bayarsaikhan M, Lu S, Gonzalez-Mera L, Sivadorai P, Nowak KJ, Ravenscroft G, Mastaglia FL, North KN, Ilkovski B, Kremer H, Lammens M, van Engelen BG, Fabian V, Lamont P, Davis MR, Laing NG, Goldfarb LG (2010) Dominant mutations in KBTBD13, a member of the BTB/Kelch family, cause nemaline myopathy with cores. Am J Hum Genet 87(6):842-847. doi:10.1016/j.ajhg.2010.10.020. Epub 2010 Nov 25. Erratum in: Am J Hum Genet. 2011 Jan 7;88(1):122

10. Ravenscroft G, Miyatake S, Lehtokari VL, Todd EJ, Vornanen P, Yau KS, Hayashi YK, Miyake N, Tsurusaki Y, Doi H, Saitsu H, Osaka H, Yamashita S, Ohya T, Sakamoto Y, Koshimizu E, Imamura S, Yamashita M, Ogata K, Shiina M, Bryson-Richardson RJ, Vaz R, Ceyhan O, Brownstein CA, Swanson LC, Monnot S, Romero NB, Amthor H, Kresoje N, Sivadorai P, et al. (2013) Mutations in KLHL40 Are a Frequent Cause of Severe Autosomal-Recessive Nemaline Myopathy. Am J Hum Genet 93(1):6-18. doi:10.1016/j. ajhg.2013.05.004. Epub 2013 Jun

11. Gupta VA, Ravenscroft G, Shaheen R, Todd EJ, Swanson LC, Shiina M, Ogata K, Hsu C, Clarke NF, Darras BT, Farrar MA, Hashem A, Manton ND, Muntoni F, North KN, Sandaradura SA, Nishino I, Hayashi YK, Sewry CA, Thompson EM, Yau KS, Brownstein CA, Yu TW, Allcock RJ, Davis MR, Wallgren-Pettersson C, Matsumoto N, Alkuraya FS, Laing NG, Beggs AH (2013) Identification of KLHL41 Mutations Implicates BTB-Kelch-Mediated Ubiquitination as an Alternate Pathway to Myofibrillar Disruption in Nemaline Myopathy. Am J Hum Genet 93(6):1108-1117. doi:10.1016/j.ajhg.2013.10.020. Epub 2013 Nov 21

12. Wallgren-Pettersson C, Sewry CA, Nowak KJ, Laing NG (2011) Nemaline myopathies. Semin Pediatr Neurol 18(4):230-238. doi:10.1016/j. spen.2011.10.004. Review

13. Donner K, Sandbacka M, Lehtokari VL, Wallgren-Pettersson C, Pelin K (2004) Complete genomic structure of the human nebulin gene and identification of alternatively spliced transcripts. Eur J Hum Genet 12(9):744-751

14. Pelin K, Wallgren-Pettersson C (2008) Nebulin-a giant chameleon. Adv Exp Med Biol 642:28-39

15. Böhm J, Vasli N, Malfatti E, Le Gras S, Feger C, Jost B, Monnier N, Brocard J, Karasoy H, Gérard M, Walter MC, Reilich P, Biancalana V, Kretz C, Messaddeq N, Marty I, Lunardi J, Romero NB, Laporte J (2013) An integrated diagnosis strategy for congenital myopathies. PLoS One 8(6):e67527. doi:10.1371/ journal.pone.0067527

16. Kiiski K, Laari L, Lehtokari VL, Lunkka-Hytönen M, Angelini C, Petty R, Hackman P, Wallgren-Pettersson C, Pelin K (2013) Targeted array comparative genomic hybridization-a new diagnostic tool for the detection of large copy number variations in nemaline myopathy-causing genes. Neuromuscul Disord 23(1):56-65. doi:10.1016/j.nmd.2012.07.007. Epub 2012 Sep 23

17. Lehtokari VL, Pelin K, Sandbacka M, Ranta S, Donner K, Muntoni F, Sewry C, Angelini C, Bushby K, Van den Bergh P, lannaccone S, Laing NG, Wallgren-Pettersson C (2006) Identification of 45 novel mutations in the nebulin gene associated with autosomal recessive nemaline myopathy. Hum Mutat 27(9):946-956

18. Romero NB, Clarke NF (2013) Congenital myopathies. Handb Clin Neurol 113:1321-1336. doi:10.1016/B978-0-444-59565-2.00004-6. Review

19. Gurgel-Giannetti J, Reed UC, Marie SK, Zanoteli E, Fireman MA, Oliveira AS, Werneck LC, Beggs AH, Zatz M, Vainzof M (2003) Rod distribution and muscle fiber type modification in the progression of nemaline myopathy. J Child Neurol 18(3):235-240

20. Fardeau M (1969) Etude d'une nouvelle observation de 'Nemaline Myopathy'. II-Données ultrastructurales. Acta Neuropathol 13:250-266

21. North KN, Laing NG, Wallgren-Pettersson C (1997) Nemaline myopathy: current concepts. The ENMC International Consortium and Nemaline Myopathy. J Med Genet 34(9):705-713. Review

22. Goebel HH (1996) Congenital myopathies. Semin Pediatr Neurol 3(2):152-161. Review

23. Ryan MM, Ilkovski B, Strickland CD, Schnell C, Sanoudou D, Midgett C, Houston R, Muirhead D, Dennett X, Shield LK, De Girolami U, lannaccone ST,
Laing NG, North KN, Beggs AH (2003) Clinical course correlates poorly with muscle pathology in nemaline myopathy. Neurology 60(4):665-673

24. Fardeau M (1982) Congenital myopathies. In: Mastaglia FL, Walton SJ (ed) Skeletal muscle pathology. Chrurchill Livingston, London, pp 161-203

25. Wallgren-Pettersson C, Rapola J, Donner M (1988) Pathology of congenital nemaline myopathy. A follow-up study. J Neurol Sci 83(2-3):243-257

26. Lehtokari VL, Greenleaf RS, DeChene ET, Kellinsalmi M, Pelin K, Laing NG, Beggs AH, Wallgren-Pettersson C (2009) The exon 55 deletion in the nebulin gene-one single founder mutation with world-wide occurrence. Neuromuscul Disord 19(3):179-181

27. Vasli N, Böhm J, Le Gras S, Muller J, Pizot C, Jost B, Echaniz-Laguna A, Laugel V, Tranchant C, Bernard R, Plewniak F, Vicaire S, Levy N, Chelly J, Mandel JL, Biancalana V, Laporte J (2012) Next generation sequencing for molecular diagnosis of neuromuscular diseases. Acta Neuropathol 124(2):273-283

28. Minobu S, Valerie B, Michel F, Jean-Yves H, Makiko O, Jocelyn L (2013) Romero Norma Beatriz Extensive morphological and immunohistochemical characterization in myotubular myopathy. Brain and Behavior 7:476-486

29. Dubowitz V, Oldfors A, Sewry C (eds): (2013) Muscle biopsy A practical approach 4th edition. Saunders Elsevier, Philadelphia

30. Farkas-Bargeton E, Diebler MF, Arsénio-Nunes ML, Wehrlé R, Rosenberg B (1977) Histochemical, quantitative and ultrastructural maturation of human fetal muscle. J Neurol Sci 31(2):245-259. French

31. Romero NB, Mezmezian M, Fidzianska A (2013) Main steps of skeletal muscle development in the human: morphological analysis and ultrastructural characteristics of developing human muscle. In: Dulac O, Lassonde M, Sarnat HB (ed) Pediatric Neurology Part III. Handbook of Clinical Neurology, vol 113 3rd series. Elsevier, Amsterdam, pp 1299-1301

32. Ottenheijm CAC, Witt CC, Stienen GJ, Labeit S, Beggs AH, Granzier H (2009) Thin filament length dysregulation contributes to muscle weakness in nemaline myopathy patients with nebulin deficiency. Hum Mol Genet 18 (13):2359-69. doi:10.1093/hmg/ddp168. Epub 2009 Apr 4

33. Ottenheijm CAC, Hooijman P, DeChene ET, Stienen GJ, Beggs AH, Granzier $H(2010)$ Altered myofilament function depresses force generation in patients with nebulin-based nemaline myopathy (NEM2). J Struct Biol 170:334-343

34. Caremani M, Dantzig J, Goldman YE, Lombardi V, Linari M (2008) Effect of Inorganic Phosphate on the Force and Number of Myosin Cross-Bridges During the Isometric Contraction of Permeabilized Muscle Fibers from Rabbit Psoas. Biophys J 95:5798-5808

35. Vasli N, Laporte J (2013) Impacts of massively parallel sequencing for genetic diagnosis of neuromuscular disorders. Acta Neuropathol 125:173-185

36. Lawlor MW, Ottenheijm CA, Lehtokari VL, Cho K, Pelin K, WallgrenPettersson C, Granzier H, Beggs AH (2011) Novel mutations in NEB cause abnormal nebulin expression and markedly impaired muscle force generation in severe nemaline myopathy. Skelet Muscle 1(1):23. doi:10.1186/2044-5040-1-23

37. de Winter JM, Buck D, Hidalgo C, Jasper JR, Malik FI, Clarke NF, Stienen GJM, Lawlor MW, Beggs AH, Ottenheijm CAC, Granzier H (2013) Troponin activator augments muscle force in nemaline myopathy patients with nebulin mutations. J Med Genet 50:383-392

38. Millevoi S, Trombitas K, Kolmerer B, Kostin S, Schaper J, Pelin K, Granzier H, Labeit S (1998) Characterization of nebulette and nebulin and emerging concepts of their roles for vertebrate Z-discs. J Mol Biol 282(1):111-123

39. Ottenheijm CA, Granzier H (2010) New insights into the structural roles of nebulin in skeletal muscle. J Biomed Biotechnol 2010:968139. doi:10.1155/2010/968139

40. Sewry CA (1998) The role of immunocytochemistry in congenital myopathies. Neuromuscul Disord 8(6):394-400

41. Wallgren-Pettersson C, Donner K, Sewry C, Bijlsma E, Lammens M, Bushby K, Giovannucci Uzielli ML, Lapi E, Odent S, Akcoren Z, Topaloğlu H, Pelin K (2002) Mutations in the nebulin gene can cause severe congenital nemaline myopathy. Neuromuscul Disord 12(7-8):674-679

42. Romero NB, Bitoun M (2011) Centronuclear myopathies. Semin Pediatr Neurol 18(4):250-256. doi:10.1016/j.spen.2011.10.006

doi:10.1186/2051-5960-2-44

Cite this article as: Malfatti et al:: Muscle histopathology in nebulin-related nemaline myopathy: ultrastrastructural findings correlated to disease severity and genotype. Acta Neuropathologica Communications 2014 2:44. 